\title{
A Hierarchy of Neuromechanical and Robotic Models of Antenna-Based Wall Following in Cockroaches
}

\author{
Jusuk Lee, Owen Y. Loh and Noah J. Cowan
}

\begin{abstract}
It may come as no surprise that a simple "planar unicycle" (or "skate") model can successfully follow a wall at high speed under PD-control. What would be surprising is that such a simple control mechanism may underly the control of one of Nature's fastest terrestrial insects, the American cockroach. For this paper, we implemented the same controller (up to scale) believed to govern cockroach wall following to successfully control two models of the cockroach: a differentialdrive mobile robot with a flexible artificial antenna and a lateral leg spring model with moving center of pressure as the control input. These physical and numerical experiments demonstrate the sufficiency of the cockroach's putative controller in a realworld setting with unmodeled effects, and suggest how the nervous system might guide leg placement in response to sensory stimuli. This hierarchy of models may prove useful in generating prescriptive hypotheses for biological testing and hence elucidating the general principles that underly sensorguided animal locomotion.
\end{abstract}

\section{INTRODUCTION}

American cockroaches (Periplaneta americana) can run up to $1.5 \mathrm{~m} / \mathrm{s}$ (or 50 body lengths $/ \mathrm{s}$ ), even changing their gait to bipedal in order to achieve high speeds [9]. When they encounter a wall, they typically run along it using their antenna to regulate their distance from the wall. They are capable of tracking a zigzagged wall of up to 25 turns/s [3]. Biologists and engineers alike are interested in understanding the fundamental mechanisms that underlie such agility. Specifically, we are interested in how cockroaches process sensory data from their antenna for control of locomotion.

One approach to this problem has been a bottom-up approach: fit kinematic data to a synaptically-interconnected gross neural network model of biologically-relevant neurons. Chapman and Webb [5] implemented such a neural circuit on a mobile robot where IR sensors were used as the robot's "antenna"; their robot exhibited an escape response followed by a wall-following response, much like that of a cockroach. However, the sheer complexity of the neural network hampers any formal mathematical analysis (e.g., stability analysis) on their neural controller. In addition, the absence of the robot's mechanical parameters (e.g., body mass and inertia) in the overall modeling process may produce synaptic weights that may not reflect the animal's actual neural controller [6].

In this paper, we use an alternative, top-down approach: find the simplest control law (in the classical control theory

O. Loh was supported by a JHU Provosts Undergraduate Research Award. J. Lee and N. Cowan are with the Mechanical Engineering Department at Johns Hopkins University, Baltimore, MD 21218 USA. \{jsl, ncowan\}@ jhu.edu

O. Loh is with the Mechanical Engineering Department at Northwestern University, Evanston, IL 60208 USA. o-loh@northwestern. edu sense) that abstractly models the cockroach's neural controller. This approach elucidates the general functions of the neural network as a whole, allowing one to predict how actual neural signals may look [6], [7]. Of course, the models we present in this paper are behavior specific, and thus do not capture the complex dynamics-such as switching between escape response and wall following - that may emerge out of more elaborated neural-network-based models [5].

We utilize the notion of templates and anchors [8] to systematically formulate the synthesis of sensory, body, and neural controller dynamics; such formulation makes the model amenable to formal mathematical analyses. A template is the simplest model that captures a specific behavior, while an anchor is a more complex, representative model of the behavior. Templates and anchors are not just "simple models" and "complex models"; there must be a natural embedding of the template behavior within the anchor [8]. For example, horizontal plane locomotion in sprawled-posture animals, such as many insects, is well characterized by the lateral leg-spring (LLS) template because the animal's center of mass (COM) bounces side-to-side [23]-[25]. In this paper, we consider multiple levels in a template-anchor hierarchy (shown in Fig. 1) and show (1) how they are "connected" to each other and (2) biological predictions one can generate from each level in the hierarchy.

In Section II, we briefly review the simplest template for high-speed wall following proposed by Cowan et al. [7]: antenna-based planar unicycle (APU) (see Fig. 1). Using classical control theory, they show that P-control is not sufficient for stable wall following. For their experiments the next simplest control law, PD-control, most parsimoniously accounts for the data.

In Section III, we consider one anchor for the APU model: our mobile robot with an artificial antenna (see Fig. 1). We first discuss the design of the antenna as well as its construction. Then we show the analytical embedding of the APU model in the Garcia robot model. Using the principle of similitude, we map the best-fit parameters from the cockroach ethological experiments to the robot's parameters and control gains; the robot exhibits stability without any parameter tuning. The success of our trials demonstrates the sufficiency of the PD-controller in a real-world setting with unmodeled effects including a flexible antenna and its friction with the wall. In addition, our result supports Camhi and Johnson's [3] claim that the sensors at the base of the cockroach antenna may not be necessary in the high-speed wall following.

In Section IV, we consider another anchor for the APU 


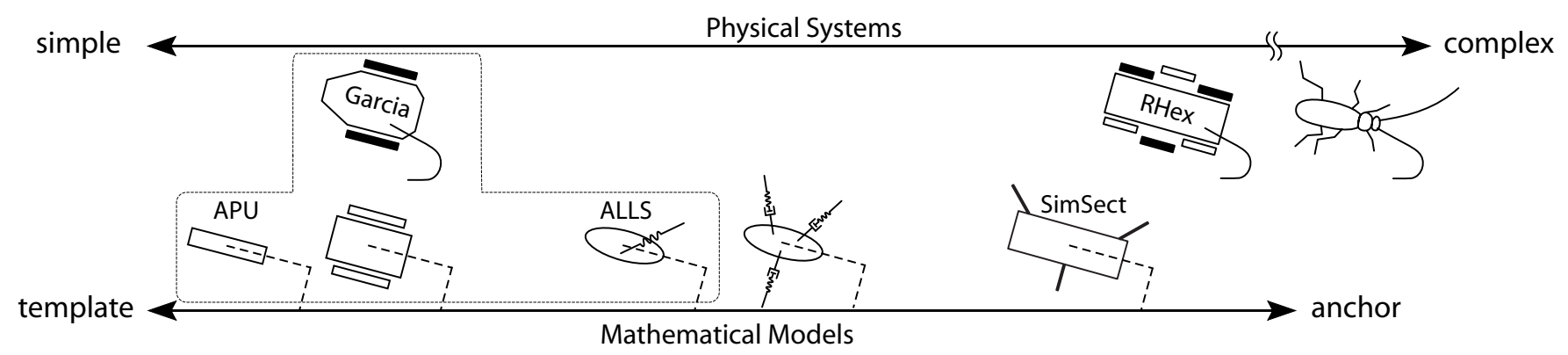

Fig. 1. Our research program involves multiple levels of modeling, robotic experimentation, ethology, and neurophysiology. In this paper, we present a sequence of antenna-based wall following models of increasing complexity, and at each stage make predictions about both robot and biological performance. Here, we present three models: the APU (Section II), a model for our Garcia robot (Section III), and the antenna-based LLS (ALLS) (Section IV). Importantly, each level of modeling admits the same control structure, including parameters (up to scale). The two physical systems involved in this study are the Garcia robot and an actual American cockroach. Eventually, we plan to extend our modeling effort to more elaborated anchors, such as the planar, multi-legged model proposed by Seipel et al. [28], or the spatial SimSect model developed by Saranli et al. [20] of RHex [21]. As well, we plan to extend our experimental robotics work to include a RHex-like six legged robot, endowed with an artificial antenna.

model: antenna-based lateral leg spring (ALLS) model (see Fig. 1). Here we also show, numerically but not formally, the embedding of the APU model within the ALLS. From this, we ask how might such a simple control mechanism, namely PD-control with an antenna, be implemented on a legged mechanism? We show that antenna-based feedback-driven placement of the center of pressure (COP) of the LLS model enables us to control the LLS to follow along a wall using a virtual antenna, with no modification of the control gains used for the lower-dimensional template model. This analysis suggests new biological experiments, and should lead to new control strategies for legged robots such as RHex [1], [21], Sprawl [4], or Whegs [19].

\section{Review: Antenna-BASEd Planar Unicycle (APU)}

In this section, we briefly review the mathematical setup by Cowan et al. [7]. They modeled the high-speed wall following of $P$. americana as a planar unicycle with polar moment of inertia, and the antenna is assumed to measure $d$ (see Fig. 2) where $d=\ell \tan \theta+y \sec \theta$.

Cowan et al. proposed the PD-control law shown in Fig. 3. This yields the closed-loop model:

$$
\begin{aligned}
\dot{y} & =v^{*} \sin \theta \\
\dot{\theta} & =\omega \\
\dot{\omega} & =-\alpha \omega \underbrace{-K_{P}\left(d-d^{*}\right)-K_{D}}_{u} \dot{d}
\end{aligned}
$$

where $\alpha=B / J, J$ is polar moment of inertia, $B$ is damping coefficient, and $u$ is the inertia-scaled polar moment that the cockroach must generate in order to turn [11]. They obtained parameters $\left(\ell, \alpha, K_{P}, K_{D}\right)$ by fitting (1) to biological highspeed wall-following data; their fitted parameter values are given in Table I (first row). Using classical control theory, Cowan et al. showed that P-control is not sufficient for stable wall following and that the next simplest control law, PDcontrol, most parsimoniously accounts for the data.

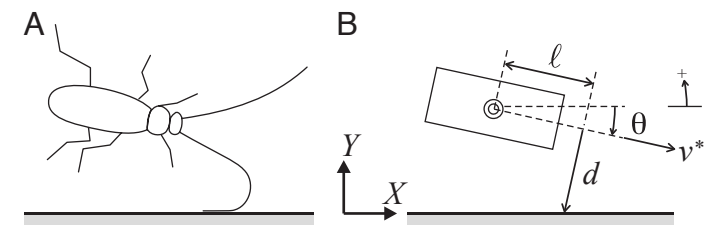

Fig. 2. (Adapted from [7]) (A) Depiction of a cockroach following a straight wall. (B) Unicycle model of the running cockroach: $\ell$ is the lookahead distance; $d$ is the antenna measurement; $v^{*}$ is the forward running speed which is assumed to be constant; $\theta$, is the angle of the cockroach body relative to the wall (note that $\theta<0$ in this figure).

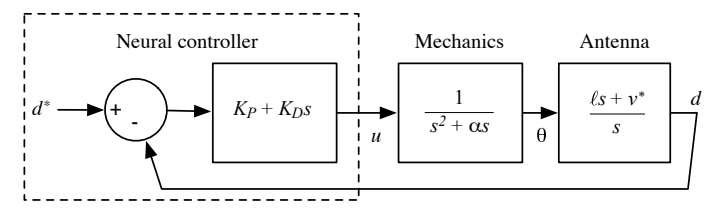

Fig. 3. (From [7]) Block diagram of simplified control model. The "mechanics" box represents the torsional dynamics. The "sensing" box is a simplified model of the antenna sensing kinematics. Cowan et al. [7] fit a simplified neural controller (in the dashed box) to their experimental data; $d^{*}$ is a nominal "desired" wall-following distance.

\section{ARtificial Antenna And Differential-Drive RовOT}

In this section, we use a mobile robot (Garcia, Fig. 5) as a test platform to support the efficacy of using the APU as a template model for the high-speed wall-following cockroach; we integrate our flexible antenna with a robot that has the same dimensionally-scaled parameters as $P$. americana. Anchoring the APU model within a physical system demonstrates the sufficiency of the PD controller suggested by Cowan et al. [7] in a real-world setting with unmodeled effects.

\section{A. Antenna Design}

Both mammalian whiskers and arthropod antennae inspired past work in robotic tactile sensing. In whiskers-an evolutionary adaptation of hairs - the length of the hair is unsensorized; thus a typical whisker-inspired probe consists of a flexible, cantilevered beam sensed solely at its base 
[12], [17], [26], [31]. Arthropod antennae, by contrast, are complex, multimodal sensory structures containing mechanical, chemical (e.g., humidity and pheromone), and thermal receptors [27], and can inspire new sensory systems in robotics [2], [14], [16].

We designed and built an artificial antenna (Fig. 4) to provide tactile feedback to our mobile robot. The design is based on our observations of real cockroaches and their antennae while wall following as well as those suggested by others [3], [22], [27], [29]. These observations and their implications toward our design are briefly summarized as follows. (1) We integrated a series of flex sensors (cut from Abrams Gentile 4" FlexSensors) along the length of the antenna in order to obtain local curvatures of the antenna; $P$. americana may also detect the bend of each segmental joint via campaniform sensilla [27]. (2) Arthropod's flagellum decreases in stiffness along its length [29], allowing the antenna to conform to a broad range of surface irregularities as it sweeps along; we tapered our antenna to give a continuously decreasing stiffness along its length. For added stiffness we added a rigid support structure in the middle of the antenna (see Fig. 5). (3) Since the flex sensors are unidirectional, we cast the tip of the antenna with a curve to prevent the tip of the antenna from bending (pointing) forward (Fig. 4(D)). (4) For the robot to predict what is coming ahead, we rigidly clamped the base of our antenna to the robot at about $35^{\circ}$ from heading; cockroaches similarly hold the base of their antenna at a relatively fixed angle during rapid wall-following (see Fig. 8(D)).

The raw data provided by the antenna consists of an amplified voltage output, $V_{i}$, from each flex sensor. To extract the distance to the wall $d$ from the voltage outputs, we performed least-squares fit. ${ }^{1}$ Here we do not consider the two proximal flex sensor measurements; the high stiffness near the base of the antenna and the addition of the support structure in the middle of the antenna have limited their motions and did not contribute significantly to the calculation of $d$. The omission of the most proximal sensory data is consistent with Camhi and Johnson's [3] experiment that distance can be encoded by distal receptors in the flagellum without the sensory data from the base.

\section{B. Review: Model of the Garcia with an Antenna}

Our physical instantiation of the unicycle-like robot is called Garcia (Acroname, Inc.) shown in Fig. 5. It is a three-wheeled robot, with two drive wheels sharing the same axis of rotation and a third passive omni-directional wheel, with zero caster sweep space, for balance. This machine was used previously by Lamperski et al. [14] to demonstrate the feasibility of wall following using a multi-rigid-link antenna as its distance sensor.

\footnotetext{
${ }^{1}$ We placed the antenna (attached to our robot) against a wall parallel to the body of the robot and recorded outputs from each flex sensor as we varied $d$. We then placed the robot at a $30^{\circ}$ angle to the wall and read the outputs from the flex sensor while we varied $d$. Finally, we applied a least-squares fit to the data to obtain the parameters $a \in \mathbb{R}^{2}, b \in \mathbb{R}$ for the affine relationship $d=a^{T} V+b$.
}

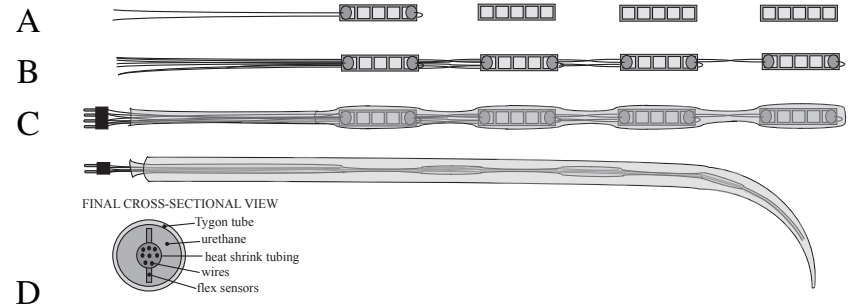

Fig. 4. An overview of the cast urethane antenna construction process (A) We cut the flex sensors into $2.5 \mathrm{~cm}$ lengths and attach wire leads to both ends of each flex sensor using conductive epoxy as shown on the leftmost gauge. (B) Next we arrange the two wires from each gauge to run behind the other gauges down to the base, ensuring they will remain within the neutral bending axis during normal operation. (C) We then slide the gauges and wires into a heat-shrink tube and shrink it leaving ample room for the wires to move freely. At the base, the wires are soldered to a single header which plugs into custom amplifiers on the robot. (D) We slide the assembly into a pre-shaped (by heating until soft, forming, then cooling) Tygon rubber tube. We fill the Tygon tube with a two-part urethane casting compound using a vacuum pump and allow it to cure for 48 hours prior to calibration. Finally we wrap Teflon tape along the antenna to reduce the friction between the antenna and the wall.

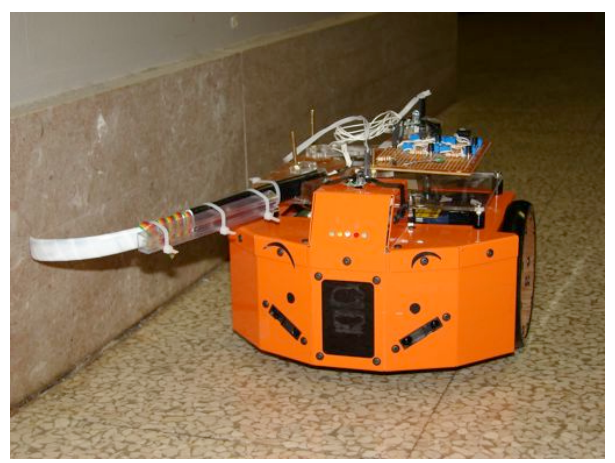

Fig. 5. The cast urethane antenna with embedded flex sensors mounted on the Garcia robot.

Following the similar characterization of the feedback stability as Lamperski et al., we have

$$
\left[\begin{array}{c}
\dot{y} \\
\dot{\theta} \\
\dot{\Sigma} \\
\dot{v} \\
\dot{\omega}
\end{array}\right]=\left[\begin{array}{c}
v \sin \theta \\
\omega \\
v-v^{*} \\
-\gamma v \underbrace{-K_{V}\left(v-v^{*}\right)-K_{I} \Sigma}_{u_{1}} \\
-\alpha \omega \underbrace{-K_{P}\left(d-d^{*}\right)-K_{D}}_{u_{2}} \dot{d}
\end{array}\right],
$$

where $\alpha$ and $\gamma$ account for the robot's electrical and mechanical parameters such as back-EMF constant and wheel moment of inertia. The only difference between (2) and that of Lamperski et al. is that they use $\omega$ instead of $\dot{d}$ in the feedback law $u_{2}$. Linearizing the system at its equilibrium point $(y, \theta, \Sigma, v, \omega)=\left(d^{*}, 0,-\alpha v^{*} / K_{I}, v^{*}, 0\right)$ reveals that the system is stable exactly when

$$
\begin{aligned}
& K_{V}>-\gamma, K_{I}>0, \\
& K_{P}>0, K_{D}>-\frac{\alpha}{\ell}, \frac{K_{D}}{K_{P}} v^{*}>\frac{v^{*}}{\alpha+K_{D} \ell}-\ell,
\end{aligned}
$$

where $\gamma, \alpha, v^{*}, \ell>0$. Notice that the conditions for forward 
TABLE I

PARAMETER VALUES FOR $P$. americana AND THE GARCIA.

\begin{tabular}{c|ccccc} 
& $\begin{array}{c}v^{*} \\
(\mathrm{~m} / \mathrm{s})\end{array}$ & $\begin{array}{c}\ell \\
(\mathrm{m})\end{array}$ & $\begin{array}{c}\alpha \\
\left(\mathrm{m}^{-1} \mathrm{~s}^{-2}\right)\end{array}$ & $\begin{array}{c}K_{P} \\
\left(\mathrm{~m}^{-1} \mathrm{~s}^{-1}\right)\end{array}$ & $\begin{array}{c}K_{D} \\
\left(\mathrm{~s}^{-1}\right)\end{array}$ \\
\hline P. americana & 0.352 & 0.027 & 12.7 & 2600 & 433 \\
Garcia & 0.5 & $\mathbf{0 . 1 3 9}$ & 3.53 & $\mathbf{3 9 . 1 6}$ & $\mathbf{2 3 . 4 9}$
\end{tabular}

stability-which are constraints on $K_{V}$ and $K_{I}$-are decoupled from the conditions on rotational stability - which are constraints on $K_{P}$ and $K_{D}$.

\section{Dynamically-Scaled Parameters of P. americana for the Garcia}

We found the necessary parameters for the Garcia using the principle of similitude; the APU's torsional dynamics equation (1) and the last row of (2) are identical, namely they are of the form

$$
\dot{\omega}=-\alpha \omega-K_{P}\left(d-d^{*}\right)-K_{D} \dot{d},
$$

where $\alpha, K_{P}$, and $K_{D}$ are known quantities for the cockroach [7]. Since we know $\alpha$ for the robot as well [14], selecting two fundamental units, $v$ and $\ell$, leads to the following dimensionless ratios:

$$
\widetilde{\alpha}=\frac{\alpha \ell}{v^{*}}, \widetilde{K}_{P}=\frac{K_{P} \ell^{3}}{v^{* 2}}, \widetilde{K}_{D}=\frac{K_{D} \ell^{2}}{v^{*}} .
$$

Setting the desired velocity for the Garcia to $0.5 \mathrm{~m} / \mathrm{s}$ and assuming $\widetilde{\alpha}$ to be constant, we calculated the dimensionallyscaled look-ahead distance for the Garcia. Then we found values for $K_{P}$ and $K_{D}$ in the similar way. The calculated values are shown in bold in Table I (second row).

\section{Experiments}

The experimental setup for the Garcia was similar to that of the cockroach behavioral experiment by Cowan et al. [7]. The robot followed a wall that consisted of a straight control wall (to allow the robot to reach its steady state) and an angled wall to act as a "step" perturbation to the internal state, $\theta$. We used the parameter values for the Garcia shown in Table I with $K_{V}=1 \mathrm{~s}^{-1}$ and $K_{I}=1 \mathrm{~s}^{-2}$. This set of parameters satisfied the stability conditions (3).

All 30 trials at $v^{*}=0.5 \mathrm{~m} / \mathrm{s}$ with the $30^{\circ}$ angled wall demonstrated successful turning (e.g., Fig. 8(B)). Our somewhat arbitrary choice of speed gains $\left(K_{V}, K_{I}\right)$ produced substantial oscillations in the forward speed (Fig. 6(A)), but nevertheless reached steady state speed within about two seconds and did not imperil wall-following performance.

From our experiments we noticed a few undesirable behaviors. First, the robot followed the wall with a constant error in $d$ (Fig. 6(B)). We believe that this was caused by the nonnegligible force produced by the antenna against the wall. One remedy to this problem would be to either introduce an integral controller or design the antenna to be less stiff (the latter solution would be more consistent with the biology). Second, the figure shows that flex sensors 3 and 4 (E, F) did not return to their original configuration after encountering
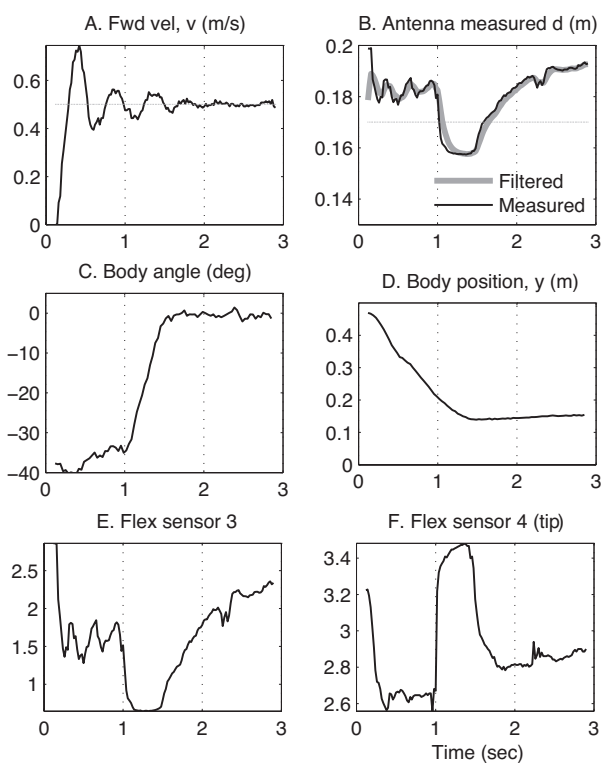

Fig. 6. The Garcia's internal states during a trial with parameters from Table I. $\left(d^{*}=0.17 \mathrm{~m}, v^{*}=0.5 \mathrm{~m} / \mathrm{s}\right)(\mathrm{B}, \mathrm{D})$ were obtained from the overhead camera images. (B) shows the raw (thin) and lightly filtered (thick) $d$ values; the Garcia used the filtered $d$ and $\dot{d}$ in (4). (E,F) show raw voltages from the respective amplifiers for flex sensors; hence, they are shown for their qualitative forms.

the angled wall. One possible explanation could be that there is a range of "stable" configurations of the antenna for a given $d$ due to friction, memory effects, etc. A reasonable calibration can cope with this as suggested by the fact that the Garcia was able to follow the wall successfully after making the turn despite the drift. Third, the Garcia failed to navigate turns of angles greater than $40^{\circ}$. We believe this problem will be addressed through the design of more flexible antennae with more appropriately tapered mechanical stiffness.

\section{E. Biological Implications from Robotics Experiments}

Experiments performed on a robotics platform can guide researchers in generating, refuting, and supporting biological hypotheses [10]. We see two such instances from our experimental result. First, the success of our trials supports Cowan et al.'s [7] work by demostrating the sufficiency of the PD-controller in a real-world setting with unmodeled effects including a flexible antenna and its friction with the wall. Our result also supports Camhi and Johnson's [3] claim that turning, in cockroach wall following, is mediated predominantly by the flagellum and not necessarily the base of the antenna; our robot controller uses only the two most distal flagellar segments of an antenna without the flex sensor data from the base of the antenna.

\section{The Antenna-BASed Lateral Leg Spring (ALLS)}

In the previous models, APU and the Garcia, the control input was literally the torque applied to the body through continuous actuation. What is less clear, however, is how might the simplest wall-following model (APU, Section II) be embedded in more elaborated anchors. Further, can we 


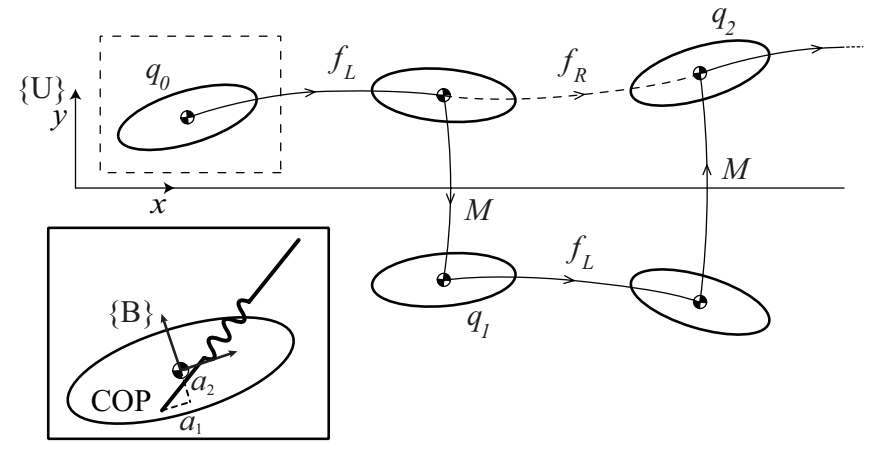

Fig. 7. (Adapted from [15]) Illustration of multi-step dynamics and its equivalent representations of the LLS model; legs are not shown for clarity. Inset: A schematic of the LLS model at the start of a left step; $\left[a_{1}, a_{2}\right]^{T}$ is the location of the COP written in $\{\mathrm{B}\}$.

glean from such an embedding how a PD signal might be applied to control stride-to-stride dynamics in a legged organism? Here we present first steps toward answering these questions by adding antenna-based control to the Lateral Leg Spring model of cockroach running.

\section{A. Review: The LLS Model}

Inspired by Kubow and Full's model [13], Schmitt and Holmes introduced the lateral leg spring model [24], [25]; it is a horizontal template model to study running sprawlpostured animals that exhibit lateral oscillations during each stride. The model is a rigid body on a frictionless surface that has two massless, spring-loaded, telescoping legs as shown in Fig. 7 (inset). The legs attach to the body at a point called center of pressure (COP) (parameterized by $a_{1}$ and $a_{2}$ ), and they can rotate freely about that point. Cockroaches run in a tripod gait; hence, in effect, each virtual leg represents the three stance legs. For ease of explanation, we only consider a left step; the right step dynamics is a reflection of the left step dynamics along the $x$-axis in $\{\mathrm{U}\}$ (Fig. 7).

At the start of a left step, the left leg affixes its foot to the ground with leg base angle $\beta_{0}$ and relaxed leg length $l_{0}$. Due to the body's initial velocity, the leg gets compressed while rotating about its foot and the COP. The compressed leg generates a counter force to the body along the leg and its attachment point to the body at the COP. As the body moves ahead of the foot contact point, the spring starts to decompress and pushes the body forward. When the left leg returns back to its relaxed length $l_{0}$, the left leg lifts off and the right leg touches down (at an angle $-\beta_{0}$ ) simultaneously, and the right step ensues.

For $a_{2}=0$, Schmitt and Holmes [24] have shown that if $a_{1}<0, \delta$ (relative heading: the direction of the COM velocity relative to the body angle) and $\omega$ (angular velocity) are asymptotically stable; if $a_{1}>0$, they are unstable; if $a_{1}=0$, they are neutrally stable. That is, they have shown that the body mechanics alone can stabilize the LLS system without any active feedback control! However, having a fixed COP on the fore-aft axis yields yaw dynamics that do not match that of actual biological data. Schmitt et al. [23] resolved the issue by moving the COP during a step. An alternative, slightly simpler solution was proposed by Lee $e t$ al. [15]: place the COP laterally offset to the side, i.e., have $a_{2}=-$ const $<0$ for a left step and $a_{2}=$ const for a right step. They have shown that the LLS model is stable even when the COP is placed in front of the COM $\left(a_{1}>0\right)$ as long the COP is sufficiently offset to the side.

For task-level control of the multi-stride dynamics, we use the notation used by Lee et al. [15] and represent discrete state evolution by

$$
q_{k+1}=f\left(q_{k}, u_{k}\right):=M f_{L}\left(q_{k}, u_{k}\right),
$$

where $M=\operatorname{diag}\{-1,-1,-1,-1\} \quad(M M=I), q_{k}=$ $(\delta, \theta, \dot{\theta}, y)_{k}^{T}$, and $u_{k}$ is the control input at $k$-th step. Here, they were able to omit states $x$ and $v$ due to translational symmetry and conservation of energy. $f_{L}$ and $f_{R}$ are maps for a left and right step, respectively (see Fig. 7). The matrix $M$ mirrors the states about the $x$-axis. Then the above definition eliminates the need to distinguish between left and right steps for control purposes.

\section{B. Antenna-Based LLS (ALLS) Control Strategy}

In this section, we discuss our approach to controlling the LLS model, and discuss how it relates to other models in our hierarchy (Fig. 1). As a preliminary control task, we chose to have the LLS follow on top of a line or a virtual "wall" that is coincident with the $x$-axis in $\{\mathrm{U}\}$.

To achieve task-level control, we use the same antenna model that measures $d$ and $\dot{d}$ as in previous sections. In addition, we found the LLS parameters that best represented the data collected for [7] of P. americana; see Fig. 8(C) caption for the the parameter values. From those assumptions, we numerically linearized the return map around a nominal equilibrium trajectory ${ }^{2}$ (denoted by an overbar $(\cdot)$ ), to obtain the local return map

$$
e_{k+1}=A e_{k}+B u_{k}, \quad z_{k}=C e_{k}
$$

where $A=(\partial f / \partial q)(\bar{q}, 0), B=(\partial f / \partial u)(\bar{q}, 0), C=$ $(\partial h / \partial q)(\bar{q}, 0), e_{k}=q_{k}-\bar{q}, z_{k}=\left[d_{k}-\bar{d}, \dot{d}_{k}-\dot{\bar{d}}\right]^{T}$, $u_{k}:=a_{1, k}$, and $h=[d, \dot{d}]^{T}$. We used $a_{1}$ rather than $a_{2}$ as our control input because the gradient of the eigenvalues is greater in the direction of $x$-axis than $y$-axis of $\{\mathrm{B}\}$ [15]; a small displacement in $a_{1}$ should give us a greater control than that of $a_{2}$. In addition, updating the control input on every step rather than throughout a step resonates with the notion that due to the inherent mechanical stability, it puts less demand on the neural feedback [8]. The Kalman rank test showed the system (7) to be both controllable and observable. $^{3}$

\footnotetext{
${ }^{2}$ We used Levenberg-Marquardt method in Matlab's fsolve function to find the equilibrium point; while fixing the state $v$ to a desired value, the function minimized the error difference of a step, $f(q)-q$. We found the step-to-step return map Jacobian, $A$, about the equilibrium point using a central difference approximation. [15]

${ }^{3}$ Lee et al. [15] controlled their LLS model using a linearized feedback controller where, unlike this paper, (1) they used the LLS parameters for the death-head cockroach (Blaberus discoidalis); (2) they assumed that cockroaches can measure the states $h=[\delta, \omega, d]^{T}$; and (3) they found the controller gains (without being biologically relevant) only to make the linearized system stable.
} 
Here we make several approximations to the ALLS model to simplify control and connect the ALLS to a simpler model in our research program (Fig. 1). The third row of the linearized discrete dynamics (7) for parameters for $P$. americana can be written as

$$
\omega_{k+1}-\omega_{k}=-(1.96) \omega_{k}-(1.07)\left(\delta_{k}-\bar{\delta}\right)+(616.5) u_{k} .
$$

The eigenvalue associated with the relative heading, $\delta$, is quite fast $\left(\lambda_{\delta}=0.24\right)$, and our simulations confirm that $\delta_{k}-\bar{\delta}$ was at least an order of magnitude smaller than the other terms during transients. So we approximate (8) by neglecting $\delta_{k}-\bar{\delta}$ and approximate $\dot{\omega} \approx\left(\omega_{k+1}-\omega_{k}\right) f_{s}$, where $f_{s}=$ $12 \mathrm{~Hz}$ (unpublished stride frequency data from [7]) is the stride frequency. Thus, we rescale the input and approximate (8) with a continuous-time system,

$$
\dot{\omega} \approx-\alpha \omega+u^{\prime},
$$

where $\alpha \approx 23.5$ and $u^{\prime} \approx(7398) u_{k}$. This equation mirrors the unicycle model (1), and despite the fairly crude approximations, the coefficient $\alpha \approx 23.5$ in the LLS approximation (9) is within the confidence intervals of the fitted parameters for $\alpha$ in cockroaches (Table I). Also note that the $u$ in (1) is a moment (scaled by inertia), whereas in the ALLS model, the control input is the COP position. Hence the coefficient multiplying the control $u_{k}$ in (9) has been absorbed into $u^{\prime}$ for comparison purposes.

The similarity between the two equations, although a rough approximation, has revealed the possibility that the same control structure, $u^{\prime}=-K_{P} d-K_{D} \dot{d}$, with the same parameters that were fitted to the cockroach and applied to the Garcia model, may be applicable to the ALLS. Using these gains have yielded closed loop system of $e_{k+1}=$ $(A+B K C) e_{k}$ with all of its eigenvalues $(-0.60 \pm j 0.10$ and $-0.23 \pm j 0.50)$ inside the unit circle.

Simulation for this controller using the parameters for $P$. americana is shown in Fig. 8(C). In this control law, COP lies nominally along the body $y$-axis, namely $a_{1}=0$ and $a_{2}=-3 \mathrm{~mm}$ (for the left step); the feedback controller varies the COP in the $a_{1}$ direction and the value for $a_{1}$ is saturated to $\pm 7 \mathrm{~mm}$.

\section{Toward a Motor Control Strategy for Sensor-Based Task- Level Maneuvering in Legged Runners}

The most parsimonious controller sufficient to stabilize high-speed wall following in the APU model is a continuous PD-controller mapping antenna measurements to a continuous moment about the COM. Above, we show that this control law applies with essentially no modification to the control of a legged running model, ALLS, by mapping sensor values to the COP position during each step. The next step from this work is to find evidence in running animals that links the actual COP motion to the sensory stimulus. The experimental paradigm would consist of cockroaches following along a wall with perturbations [3], [7], while individual leg forces and kinematics are measured to recover COP motions [30]. Together, these data can be used to approximate the mapping from antennal measurements to COP motions. From the above modeling, we hypothesize that the motion of the COP from step to step is governed by a PD controller, whose parameters are given in Table I. Looking further, increasingly anchored models which represent cockroach kinematics with increasing biofidelity can be used to tease apart the contributions of individual legs during turning [11], again within our template-anchor research program. With this biological understanding, we will be poised to create bioinspired control strategies for hexapedal robots that shift the COP based on sensory stimuli.

\section{CONCLUSion}

Our research program (Fig. 1) integrates models and experiments at several levels of complexity, from the simple 3 degree-of-freedom (DOF) APU, up to the animal itself with orders of magnitude more mechanical DOFs. The result is a multifaceted view (Fig. 8) of one of the most extraordinary reflex-driven locomotor behaviors ever studied [3]: high-speed antenna-based wall following in the American cockroach.

While it may come as no surprise that a wheeled robot can successfully follow a wall at high speed under PDcontrol, it is surprising that such a simple control mechanism may underly the control of one of Nature's fastest terrestrial insects [18]. This paper presents evidence from several levels of mathematical models and robotics experiments that just such a mechanism may be in place. Along the way, we constructed a cockroach-inspired tactile sensor that can be used for new robotics applications, and to test new biological hypotheses. Future iterations of this reciprocal inspiration of biology, modeling, and robotics promise to continue elucidating the principles that underly biological control, and lead to the design of dynamic, maneuverable legged robots.

\section{ACKNOWLEDGMENTS}

Thanks to Brett Kutscher for help with software and hardware integration on the Garcia robot. Thanks to Simon Sponberg, Robert Full and Andrew Lamperski for their comments on the manuscript. Thanks to John Schmitt for providing significant insight into the subtleties of the Lateral Leg Spring model.

\section{REFERENCES}

[1] R. Altendorfer, D. E. Koditschek, and P. Holmes. Stability analysis of a clock-driven rigid-body slip model for rhex. The International Journal of Robotics Research, 2004.

[2] T. Barnes, T. Truong, G. Adams, and N. McGruer. Large deflection analysis of a biomimetic lobster robot antenna due to contact and flow. Transactions of the ASME, 68:948-951, 2001.

[3] J. M. Camhi and E. N. Johnson. High-frequency steering maneuvers mediated by tactile cues: antenna wall-following in the cockroach. $J$ Exp Bio, 202:631-643, 1999.

[4] J. G. Cham, S. A. Bailey, J. E. Clark, R. J. Full, and M. R. Cutkosky. Fast and robust: Hexapedal robots via shape deposition manufacturing. The International Journal of Robotics Research, 21(10), 2002.

[5] T. P. Chapman and B. Webb. A model of antennal wall-following and escape in the cockroach. J Comp Physiol A Neuroethol Sens Neural Behav Physiol, 192(9):949-969, Sep 2006.

[6] N. J. Cowan and E. S. Fortune. The critical role of locomotion dynamics in decoding sensory systems. J Neurosci, 27(5):1123-1128, 2007. 

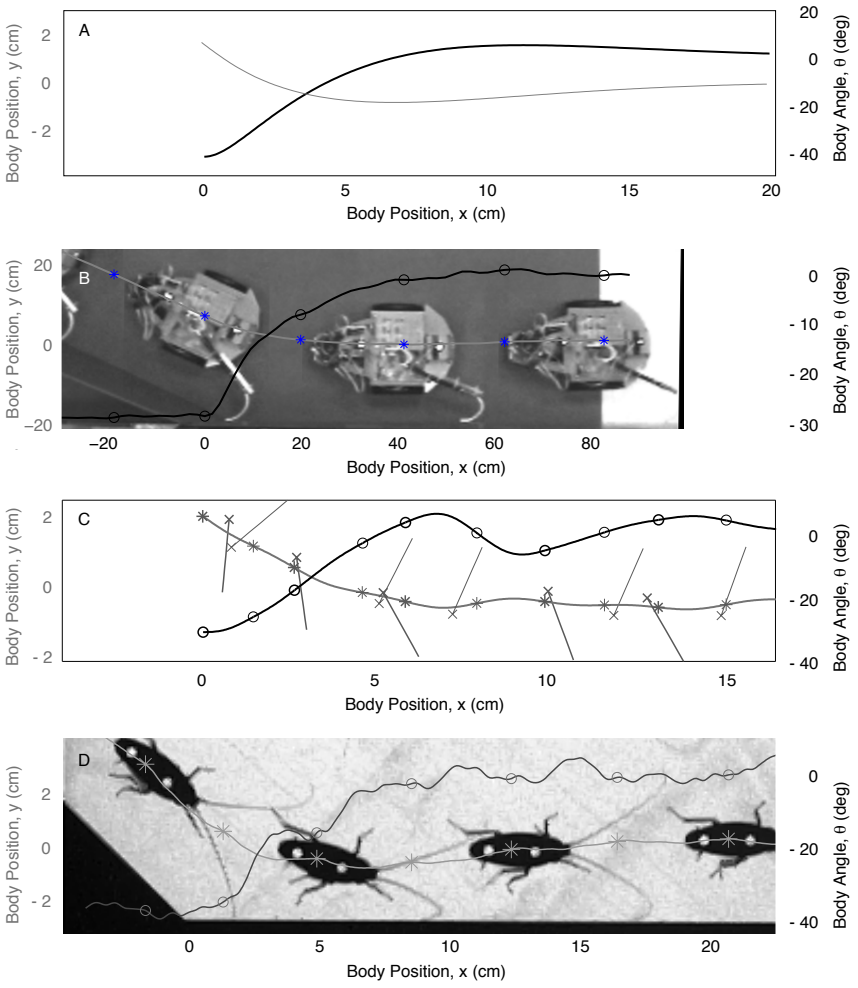

Fig. 8. Subplots (A-D) show the task-space trajectories of several models and physical systems from our research program (Fig. 1), all with approximately dimensionally equivalent parameters, and the same PD control law in each case: (A) APU model; (B) the Garcia with antenna, where the robot is shown every other "stride"; (C) the ALLS model shown at the start of every step with *'s indicating COM, $\times$ 's indicating COP; the straight lines emanating from $\times$ 's depicts LLS's leg; (D) a typical trial of $P$. americana (the cockroach is shown every other stride). For (C): $m=0.77 \times 10^{-3} \mathrm{~kg}, J=1.0 \times 10^{-7} \mathrm{kgm}^{2}, l_{0}=0.021 \mathrm{~m}$, $k=0.944 \mathrm{~N} / \mathrm{m}, \beta_{0}=1.138 \mathrm{rad}, a_{1}=0 \mathrm{~m}, a_{2}=-0.003 \mathrm{~m}$, $v(0)=0.425 \mathrm{~m} / \mathrm{s}$.

[7] N. J. Cowan, J. Lee, and R. J. Full. Task-level control of rapid wall following in the American cockroach. J Exp Bio, 209(9):1617-1629, 2006.

[8] R. J. Full and D. E. Koditschek. Templates and anchors: neuromechanical hypotheses of legged locomotion on land. J Exp Bio, 202(23):3325-3332, 1999.

[9] R. J. Full and M. S. Tu. Mechanics of a rapid running insect: two-, four-, and six-legged locomotion. J Exp Bio, 156:215-231, 1991.

[10] P. Holmes, R. J. Full, D. Koditschek, and J. Guckenheimer. The dynamics of legged locomotion: Models, analyses, and challenges. SIAM Review, 48(2):207-304, 2006.

[11] D. L. Jindrich and R. J. Full. Many-legged maneuverability: Dynamics of turning in hexapods. J Exp Bio, 202(12):1603-1623, 1999.

[12] M. Kaneko, N. Kanayama, and T. Tsuji. Active antenna for contact sensing. IEEE Transactions on Robotics and Automation, 14(2):278$291,1998$.
[13] T. M. Kubow and R. J. Full. The role of the mechanical system in control: a hypothesis of self-stabilization in hexapedal runners. Philosophical Transactions of the Royal Society of London Serieseoleol B-Biological Sciences, 354(1385):849-861, 1999.

[14] A. Lamperski, O. Loh, B. Kutscher, and N. J. Cowan. Dynamical wall-following for a wheeled robot using a passive tactile sensor. In International Conference on Robotics and Automation, pages 38383843, 2005

[15] J. Lee, A. Lamperski, J. Schmitt, and N. J. Cowan. Task-Level Control of the Lateral Leg Spring Model of Cockroach Locomotion, volume 340 of Lecture Notes in Control and Information Sciences. Heidelberg: Springer-Verlag, 2006.

[16] W. A. Lewinger, C. M. Harley, R. E. Ritzmann, M. S. Branicky, and R. D. Quinn. Insect-like antennal sensing for climbing and tunneling behavior in a biologically-inspired mobile robot. In IEEE International Conference on Robotics and Automation, 2005.

[17] M. Lungarella, V. V. Hafner, R. Pfeifer, and H. Yokoi. An artificial whisker sensor for robotics. In Proceedings of the 15th IEEE/RSJ International Conference on Intelligent Robots and Systems (IROS), pages 2931-2936, Lausanne, Switzerland, 2002.

[18] T. M. Merritt. Book of Insect Records, chapter Fastest Runner. University of Florida, 1999. http://ufbir.ifas.ufl.edu/.

[19] R. D. Quinn, G. M. Nelson, R. J. Bachmann, D. A. Kingsley, J. T. Offi, T. J. Allen, and R. E. Ritzmann. Parallel complementary strategies for implementing biological principles into mobile robots. The International Journal of Robotics Research, 22:169-186, 2003.

[20] U. Saranli. SimSect hybrid dynamical simulation environment. Technical Report CSE-TR-436-00, UM, Ann Arbor, MI, 2000.

[21] U. Saranli, M. Buehler, and D. E. Koditschek. RHex: A simple and highly mobile hexapod robot. International Journal of Robotics Research, 20(7):616-631, 2001.

[22] R. Schafer and T. V. Sanchez. Antennal sensory system of the cockroach, Periplaneta americana: postembryonic development and morphology of the sense organs. J. Comp. Neurol., 149:335-354, 1973

[23] J. Schmitt, M. Garcia, R. C. Razo, P. Holmes, and R. J. Full. Dynamics and stability of legged locomotion in the horizontal plane: a test case using insects. Biol Cybern, 86:343-353, 2002.

[24] J. Schmitt and P. Holmes. Mechanical models for insect locomotion: dynamics and stability in the horizontal plane I. Theory. Biol Cybern, 83:501-515, 2000.

[25] J. Schmitt and P. Holmes. Mechanical models for insect locomotion: dynamics and stability in the horizontal plane-II. Application. Biol Cybern, 83:517-527, 2000

[26] A. E. Schultz, J. H. Solomon, M. A. Peshkin, and M. J. Hartmann. Multifunctional whisker arrays for distance detection, terrain mapping, and object feature extraction. In IEEE International Conference on Robotics and Automation, 2005.

[27] G. Seelinger and T. R. Tobin. The American Cockroach, chapter Sense Organs, pages 217-245. Chapman and Hall Ltd, 11 New Feetter Lane, London EC4P 4EE, 1982.

[28] J. Seipel, P. Holmes, and R. Full. Dynamics and stability of insect locomotion: a hexapedal model for horizontal plane motions. Biol Cybern, 91(2):76-90, 2004.

[29] E. Staudacher, M. Gebhardt, and V. Dürr. Antennal movements and mechanoreception: Neurobiology of active tactile sensors. Advances in Insect Physiology, 32:49-205, 2005.

[30] L. H. Ting, R. Blickhan, and R. J. Full. Dynamic and static stability in hexapedal runners. J Exp Biol, 197:251-269, 1994.

[31] N. Ueno, M. Svinin, and M. Kaneko. Dynamic contact sensing by flexible beam. IEEE/ASME Transactions on Mechatronics, 3(4):254263, 1998. 